\title{
Efficacy of pulse steroid therapy in patients critically ill with COVID-19
}

\author{
Gundogdu O, Demir B, Coskun FO, Ersan I \\ Department of Anesthesiology and Reanimation, State Hospital, Sivas, Turkey. \\ droguzgundogdu@gmail.com
}

\section{ABSTRACT}

OBJECTIVES: The aim of this study was to determine the efficacy of pulse steroid therapy administered to patients critically ill with COVID-19 progressing into severe pneumonia.

METHODS: A total of 600 patients included in this retrospective study were divided into three groups. Group 1 (control group): 200 patients who did not receive steroid treatment, Group 2: 200 patients who received dexamethasone 1x8 milligram (mg) or methylprednisolone 1x80 mg, Group 3: (pulse steroid therapy group): 200 patients who received $1 \mathrm{~g}$ methylprednisolone followed by $1 \times 80 \mathrm{mg}$ methylprednisolone. Demographic and laboratory data were recorded.

RESULTS: Mortality rates in groups 1, 2 and 3 were $77 \%, 53.55 \%$, and $58.5 \%$, respectively. The ratios of intubated patients in groups 1,2 and 3 were $70 \%, 45.5 \%$ and $56 \%$, respectively. The numbers of patients whose D-dimer values were above $2,250 \mathrm{ng} / \mathrm{mL}$ (cut-off value for D-dimer in this study) in groups 2, 1 and 3 were 65,107 , and 105 , respectively.

CONCLUSION: Pulse steroid therapy does not shorten the duration of hospital stay, does not reduce the need for intubation and increases the risk of thrombosis by significantly increasing the level of D-dimer among patients critically and severely ill with COVID-19 (Tab. 4, Fig. 3, Ref. 20). Text in PDF www.elis.sk KEY WORDS: COVID-19, pulse steroid therapy, thrombosis, d-dimer, corticosteroid.

\section{Introduction}

The novel coronavirus disease induced by SARS-CoV2 virus is named COVID-19 and was announced as a pandemic by the World Health Organization on March 11, 2020. The diagnosis is based on polymerase chain reaction (PCR) test and computed tomography (1). Various antiviral (favipiravir, remdesivir, hydroxychloroquine), and anti-inflammatory drugs (steroids, tocilizumab, anakinra), as well as anticoagulants and fibrinolytics are used in the treatment of COVID-19 disease $(2,3)$.

The characteristics of COVID-19 progressing into severe pneumonia are increased oxygen need and increased c-reactive protein (CRP). This situation is interpreted as a result of a possible cytokine storm syndrome (CSS) (4). A progressive inflammatory process with a progressing decrease in the number of lymphocytes and increase in the number of neutrophils plays an important role in the pathogenesis of COVID-19. Thus, neutrophil/lymphocyte ratio (NLR) has an important place in the prognosis.

Pathological hyperactivity in the immune system characterized by cytokines and chemokines that are excessively secreted from immune system cells due to the uncontrolled activation caused

$\overline{\text { Department of Anesthesiology and Reanimation, State Hospital, Sivas, }}$ Turkey

Address for correspondence: O. Gundogdu, Department of Anesthesiology and Reanimation, Sivas State Hospital, 58140 Sivas, Turkey.

Phone: +90.554 .5945469$ by cytokines in inflammation focused on immune cells is called cytokine storm syndrome. High levels of interleukins (IL) such as IL-1 $\beta$, IL-1RA, IL-6, IL-8, IL-9, IL-10, IL-17, vascular endothelial growth factor (VEGF), tumor necrosis factor alpha (TNF- $\alpha$ ), pro-inflammatory chemokines and cytokines were observed in COVID-19 patients. CSS increases the risks of acute respiratory distress syndrome (ARDS) and multiple organ failure (5). Excessive hypercoagulability and microvascular thrombosis seen in the pulmonary vascular bed due to CSS in COVID-19 patients are also responsible for morbidity and mortality.

One of the treatment modalities commonly used in CSS are glucocorticosteroids (GCC). A systematic review including 25 protocols and 41 studies has shown that GCCs are used in different doses and protocols in CSS induced by COVID-19 (6). However, glucocorticosteroids may increase the risk of insulin resistance, cardiovascular disease, and bacterial infection ( 7 , $8,9)$. The most dangerous side effect of GCCs are thrombotic and thromboembolic complications that may cause multiple organ failure, which is included in the pathogenesis of COVID-19 disease and a bad prognosis criterion. Especially heparin with low molecular weight and anticoagulants should be added to the treatment protocol in the treatment of patients with COVID-19 progressing into severe pneumonia to prevent these complications. Steroid-induced myopathy is another side effect of a 3-4week corticosteroid treatment (10). Myopathy and severe muscle atrophies become a major problem in a prolonged treatment of COVID-19 (11). 


\section{3-798}

The aim of this study was to determine the efficacy of pulsesteroid therapy administered to patients hospitalized in an intensive care unit (ICU) with COVID-19 progressing into severe pneumonia or ARDS.

\section{Method}

This retrospective, single-center study included patients who were hospitalized in the intensive care unit of our hospital between April 1, 2020 and February 1, 2021, who were aged older than 18 years, tested positive with the COVID-19 polymerase chain reaction test (COVID-19 PCR), and had pneumonia involvement in at least 4 lung lobes on radiological imaging. The approval of the local ethical committee was obtained for the study. The permission to access the data of the patients was obtained from the surviving patients and relatives of deceased patients.

The data of 1,271 patients who were treated in the intensive care were screened. A total of 600 patients included in the study were divided into three groups. Group 1 (control group): 200 patients who did not receive steroid treatment, Group 2: 200 patients who received dexamethasone $1 \mathrm{x} 8$ milligram $(\mathrm{mg})$ or methylprednisolone 1x80 mg, Group 3: 200 patients who received $1 \mathrm{~g}$ methylprednisolone followed by 1 x $80 \mathrm{mg}$ methylprednisolone. Patients who tested negative with the COVID-19 PCR test, were treated with steroids for a shorter period than 4 days, received another anti-inflammatory or anti-cytokine drug (tocilizumab, anakinra) or intravenous immunoglobulin during the steroid treatment in the first four days of the intensive care stay, received methylprednisolone with a dose different from the pulse steroid therapy dose of $1 \mathrm{~g}$ for 3 days were excluded from the study. Blood, urine, and sputum cultures of all patients included in the study were collected on the first day of hospitalization. Accordingly, patients who had a secondary infection other than COVID-19 during the hospitalization in the intensive care were excluded from the study. All patients included in the study received the same antiviral treatment consisting of favipiravir in the maximal tolerable dose of $2 \times 1,600$ $\mathrm{mg}$ and maintenance dose of $2 \times 200 \mathrm{mg}$.

The age, sex, comorbidities, length of stay in ICU, state of leaving the ICU (dead or alive), need for non-invasive ventilation (NIV) or intubation during the intensive care stay, need for additional anticytokine drugs such as tocilizumab or anakinra after the first 4 days of steroid treatment, and the fact whether there was bacteria reproduction in the blood and sputum culture during the intensive care stay were recorded. C-reactive protein (CRP), D-dimer, ferritin, neutrophil count, lymphocyte count, neutrophil/lymphocyte ratio, platelet count, glucose, creatinine values in the blood samples taken from the patients in Group 2 and Group 3 before the administration of steroid treatment and on the 4th day of the steroid treatment were recorded. The same laboratory values of the Group 1 were recorded based on the blood samples taken on the first day of the intensive care admission and on the 4th day of the intensive care stay.

\section{Statistical methods}

Data obtained were statistically analyzed using SPSS (Ver: 25) computer program. Mean, standard deviation, and median values
Tab. 1. Demographic parameters and some clinical features.

\begin{tabular}{llcc}
\hline \multirow{2}{*}{ Gender } & & Number & $\%$ \\
\hline \multirow{2}{*}{ Comorbidity } & Female & 247 & 41.17 \\
& Male & 353 & 58.83 \\
\hline \multirow{2}{*}{ Ventilation support } & Absent & 114 & 19.00 \\
& Present & 486 & 81.00 \\
\hline \multirow{2}{*}{ Additional anti-cytokine use } & Mask or nasal O2 & 151 & 25.17 \\
& Non-invasive ventilation & 106 & 17.67 \\
& Intubation & 343 & 57.17 \\
\hline \multirow{2}{*}{ Bacterial growth } & Absent & 566 & 94.33 \\
& Present & 34 & 5.67 \\
\hline
\end{tabular}

were used to present descriptive analyses. Categorical variables were compared using Pearson Chi-Square Test. The changes in the values measured were examined between the groups using repeated measures analysis. ROC analysis was performed to determine the cut-off value for significant values of D-dimer and NLR parameters. The results with a $p$ value below 0.05 were considered statistically significant.

\section{Results}

Of the participants, 247 were female and 353 were male. While 486 had a comorbidity, 144 did not have any comorbidities. Of the patients, 343 needed an intubation while 106 needed NIV. While 34 patients needed anti-cytokine drugs after a 4-day steroid treatment, 566 did not need it. Bacterial reproduction was observed in 173 patients under steroids while no reproduction was observed in 427 patients (Tab. 1).

The mean age of the patient group without steroid treatment (Group 1) was $73.77 \pm 10.18$ years, and the mean duration of hospital stay in living patients was $12.87 \pm 5.84$ days. The mean $\mathrm{CRP}$ value was $136.25 \pm 94.64 \mathrm{mg} / \mathrm{L}$ on the 1 st day and $155.78 \pm$ $102.65 \mathrm{mg} / \mathrm{L}$ on the 4 th day. The mean D-dimer value was 2550.16 $\pm 3187.02 \mathrm{ng} / \mathrm{mL}$ on the $1 \mathrm{st}$ day and $3504.33 \pm 3595.43 \mathrm{ng} / \mathrm{mL}$ on the 4th day. The mean NLR value was $17.84 \pm 16.42$ on the 1 st day and $30.65 \pm 44.05$ on the 4 th day.

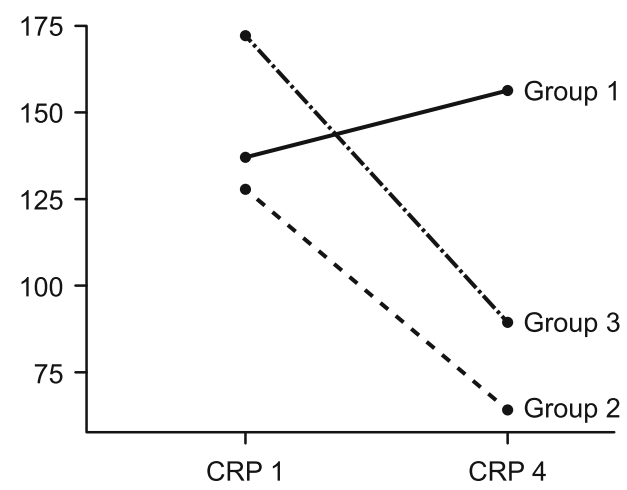

Fig. 1. Comparing the CRP changes between groups. 


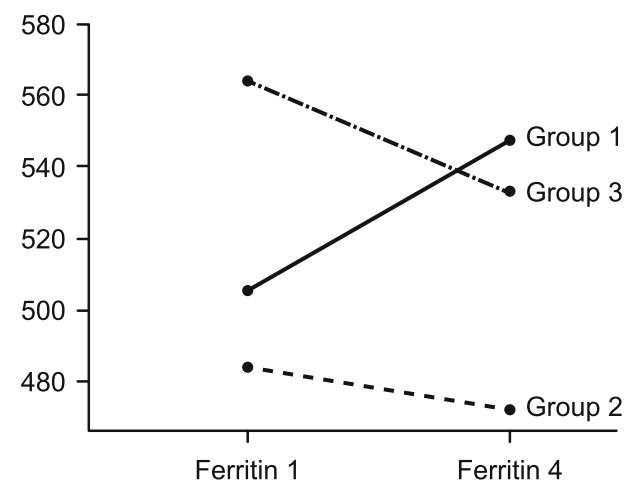

Fig. 2. Comparing the ferritin changes between groups.

The mean age of the patients in Group 2 was $7133 \pm 11.10$ years, and the mean duration of hospital stay in living patients was $11.54 \pm 5.98$ days. The mean CRP value of the patients in Group 2 was $127.13 \pm 87.48 \mathrm{mg} / \mathrm{L}$ on the 1 st day and $63.72 \pm 53.29 \mathrm{mg} / \mathrm{L}$ on the 4th day. The mean D-dimer value was $2052.98 \pm 2454.38$ $\mathrm{ng} / \mathrm{mL}$ on the 1 st day and $2199.94 \pm 2540.79 \mathrm{ng} / \mathrm{mL}$ on the 4 th day. The mean NLR value was $19.59 \pm 16.46$ on the 1 st day and $24.47 \pm 24.84$ on the 4 th day.

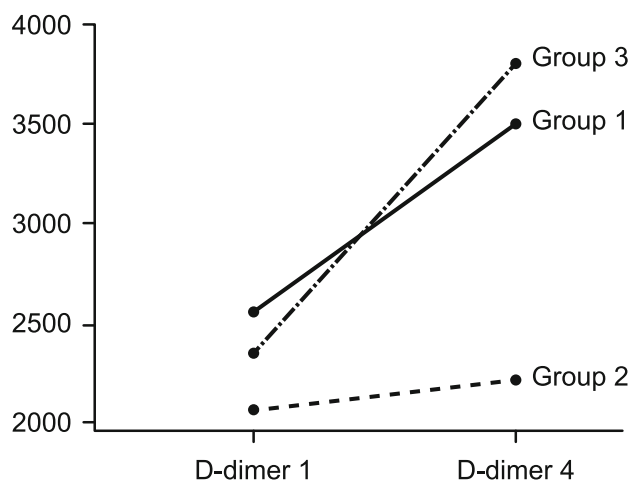

Fig. 3. Comparing the d-dimer changes between groups.

The mean age of the patients in Group 3 who received $1 \mathrm{~g}$ of methylprednisolone followed by 1 x $80 \mathrm{mg}$ of methylprednisolone for 3 days was $66.87 \pm 12.24$ years, and the mean duration of hospital stay in living patients was $12.06 \pm 6.85$ days. The mean CRP value of the patients in Group 3 was $171.19 \pm 110.47 \mathrm{mg} / \mathrm{L}$ on the 1 st day and $89.39 \pm 71.49 \mathrm{mg} / \mathrm{L}$ on the 4 th day. The mean D-dimer value was $2339.91 \pm 2772.75 \mathrm{ng} / \mathrm{mL}$ on the 1 st day and $3806.64 \pm 4029.60 \mathrm{ng} / \mathrm{mL}$ on the 4 th day. The mean NLR val-

Tab. 2. Comparing the changes in laboratory parameters between groups.

\begin{tabular}{|c|c|c|c|c|c|c|c|}
\hline & \multicolumn{2}{|c|}{ Group 1} & \multicolumn{2}{|c|}{ Group 2} & \multicolumn{2}{|c|}{ Group 3} & \multirow[t]{2}{*}{$\mathrm{p}$} \\
\hline & Mean & SD & Mean & $\mathrm{SD}$ & Mean & SD & \\
\hline Creatinine* $(\mathrm{mg} / \mathrm{dL})$ & 1.74 & \pm 1.79 & 1.42 & \pm 1.22 & 1.14 & \pm 1.04 & \multirow{2}{*}{0.044} \\
\hline Creatinine ${ }^{* *}(\mathrm{mg} / \mathrm{dL})$ & 1.81 & \pm 1.73 & 1.31 & \pm 1.21 & 1.33 & \pm 1.41 & \\
\hline$\overline{\mathrm{CRP}} *(\mathrm{mg} / \mathrm{L})$ & 136.25 & \pm 94.64 & 127.13 & \pm 87.48 & 171.19 & \pm 110.47 & \multirow{2}{*}{$<0.001$} \\
\hline $\mathrm{CRP}^{* *}(\mathrm{mg} / \mathrm{L})$ & 155.78 & \pm 102.65 & 63.72 & \pm 53.29 & 89.39 & \pm 71.49 & \\
\hline Ferritin* $(\mu \mathrm{g} / \mathrm{L})$ & 505.03 & \pm 233.77 & 483.86 & \pm 208.54 & 563.50 & \pm 278.94 & \multirow{2}{*}{$<0.001$} \\
\hline Ferritin** $(\mu \mathrm{g} / \mathrm{L})$ & 546.70 & \pm 207.64 & 471.80 & \pm 199.18 & 532.42 & \pm 191.91 & \\
\hline D-dimer* (ng/mL) & 2550.16 & \pm 3187.02 & 2053.98 & \pm 2454.38 & 2339.91 & \pm 2772.75 & \multirow{2}{*}{0.001} \\
\hline D-dimer** (ng/mL) & 3504.33 & \pm 3595.43 & 2199.94 & \pm 2540.79 & 3806.64 & \pm 4029.60 & \\
\hline Platelet* & 243.09 & \pm 122.27 & 253.28 & \pm 103.00 & 257.28 & \pm 93.35 & \multirow{2}{*}{0.005} \\
\hline Platelet** & 234.37 & \pm 123.13 & 275.22 & \pm 122.87 & 278.60 & \pm 129.11 & \\
\hline
\end{tabular}

Repeated Measurement Analysis, $\mathrm{p}<0.05$ : significant, Crp: c-reactive protein SD: standard deviation, *: day 1 parameters, **: day 4 parameters

Tab. 3. Comparing the demographic and some clinical features between groups.

\begin{tabular}{|c|c|c|c|c|c|c|c|c|}
\hline & & \multicolumn{2}{|c|}{ Group 1} & \multicolumn{2}{|c|}{ Group 2} & \multicolumn{2}{|c|}{ Group 3} & \multirow{2}{*}{$\mathrm{p}$} \\
\hline & & $\mathrm{n}$ & $\%$ & $\mathrm{n}$ & $\%$ & $\mathrm{n}$ & $\%$ & \\
\hline \multirow{2}{*}{ Gender } & Female & 93 & 46.50 & 89 & 44.50 & 65 & 32.50 & \multirow{2}{*}{0.009} \\
\hline & Male & 107 & 53.50 & 111 & 55.50 & 135 & 67.50 & \\
\hline \multirow{2}{*}{ Comorbidity } & Absent & 33 & 16.50 & 27 & 13.50 & 54 & 27.00 & \multirow{2}{*}{0.001} \\
\hline & Present & 167 & 83.50 & 173 & 86.50 & 146 & 73.00 & \\
\hline \multirow{2}{*}{ State of leaving the ICU } & Alive & 46 & 23.00 & 93 & 46.50 & 83 & 41.50 & \multirow{2}{*}{$<0.001$} \\
\hline & Dead & 154 & 77.00 & 107 & 53.50 & 117 & 58.50 & \\
\hline \multirow{3}{*}{ Ventilation support } & Mask-Nasal $\mathrm{O}_{2}$ & 46 & 23.00 & 64 & 32.00 & 41 & 20.50 & \multirow{3}{*}{$<0.001$} \\
\hline & NIV & 14 & 7.00 & 45 & 22.50 & 47 & 23.50 & \\
\hline & Intubation & 140 & 70.00 & 91 & 45.50 & 112 & 56.00 & \\
\hline \multirow{2}{*}{ Additional anti-cytokine use } & Absent & 196 & 98.00 & 197 & 98.50 & 173 & 86.50 & \multirow{2}{*}{$<0.001$} \\
\hline & Present & 4 & 2.00 & 3 & 1.50 & 27 & 13.50 & \\
\hline \multirow[t]{2}{*}{ Bacterial growth } & Absent & 141 & 70.50 & 134 & 67.00 & 152 & 76.00 & \multirow{2}{*}{0.134} \\
\hline & Present & 59 & 29.50 & 66 & 33.00 & 48 & 24.00 & \\
\hline
\end{tabular}

Chi-square test, $\mathrm{n}$ : Number, ICU: intensive care unit, NIV: non-invasive ventilation, $\mathrm{p}<0.05$ : significant 
ue was $19.71 \pm 16.71$ on the 1 st day and $31.43 \pm 52.04$ on the 4 th day.

Glucose, creatinine, CRP, ferritin, Ddimer, neutrophil, lymphocyte, NLR and platelet values measured on the 1 st and 4 th days were compared between three groups. There was a significant difference between the groups in terms of creatinine, CRP (Fig. 1), ferritin (Fig. 2), D-dimer (Fig. 3), and platelet values $(p<0.05)$. When comparing the creatinine values on the 1 st and 4 th days, the creatinine value increased from $1.74 \mathrm{mg} / \mathrm{dL}$ to $1.81 \mathrm{mg} / \mathrm{dL}$ in Group 1, decreased from $1.42 \mathrm{mg} / \mathrm{dL}$ to $1.31 \mathrm{mg} / \mathrm{dL}$ in Group 2, and increased from $1.14 \mathrm{mg} / \mathrm{dL}$ to $1.33 \mathrm{mg} / \mathrm{dL}$ in Group 3 ( $\mathrm{p}<0.05$ ).

The mean platelet value in Group 1 was $243.09 \times 10^{3}$ on the 1 st day and $234.37 \times 10^{3}$ on the 4 th day. The mean platelet value in Group 2 was $253.28 \times 10^{3}$ on the 1 st day and $471.80 \times 10^{3}$ on the 4th day. The mean platelet value in Group 3 was $257.28 \times 10^{3}$ on the 1 st day and $278.60 \times 10^{3}$ on the 4 th day $(\mathrm{p}<0.05)$.

The intergroup analyses of the laboratory parameters on the 1 st and 4th days are presented in Table 2.

The three groups were compared in terms of sex, comorbidity, the state of leaving the intensive care, type of ventilation support, necessity of additional anti-cytokine drugs, and bacterial reproduction. The ratios of male and female sexes were found to be $46.5 \%$ and $53.3 \%$ in Group 1, respectively, $44.5 \%$ and $55.5 \%$ in Group 2 , respectively, and $32.5 \%$ and $67.5 \%$ in Group 3, respectively. The presence of comorbidity was $83.5 \%$ in Group $1,68.5 \%$ in Group 2, and $73 \%$ in Group 3. While $77 \%$ of the patients in Group 1 died, the mortality rate was $53.55 \%$ in Group 2 and $58.5 \%$ in Group 3. As to the type of ventilation support, $70 \%, 45.5 \%$ and $56 \%$ of patients in groups 1, 2 and 3 were intubated, respectively. The number of patients who needed the administration of tocilizumab or anakinra after the 4-day treatment was higher in Group 3 as compared to other groups (Tab. 3 ).

The predictability of D-dimer and NLR values relative to mortality was examined with ROC analysis, and the cut-off value was determined for significant results. Considering the D-dimer cut-off value of 2,250 ng/mL, sensitivity specificity, PPD and NPD were $38.62 \%, 74.77 \%, 72.28 \%$, and $41.71 \%$, respectively. Regarding the NLR cut-off value 9.75 , sensitivity, specificity, PPD and NPD were $74.60 \%, 36.94 \%, 66.82 \%$ and $46.07 \%$, respectively. When the groups were compared based on these cut-off values for D-dimer and NLR values on the 4th day of the treatment, the numbers of patients whose D-dimer values were above 2,250 ng/ $\mathrm{mL}$ in groups 2,1 and 3 were 65,107 , and 105 , respectively. The number of patients with the NLR value above 9.75 was 158 in Group 1, 151 in Group 2, and 172 in Group 3 (Tab. 4).

\section{Discussion}

Although corticosteroids are commonly used in the treatment of COVID-19, there are ongoing discussions on the necessary dose to be applied and pulse-steroid practices with these drugs. The groups in this retrospective study were tried to be homogenized as much as possible. The differences between two treatment groups receiving corticosteroids at different doses (Group 2 and Group 3 ) and the control group (Group 1) without a corticosteroid treatment were revealed. It is believed that the results obtained in this study will change the perspective of clinicians about pulse steroid therapy in COVID-19 patients.

The increase in thrombin production and decrease in fibrinolysis due to endothelial cell dysfunction induced by severe infections like COVID-19 are observed (12). The increase in blood viscosity and hypoxia-dependent transcription due to hypoxia induced by COVID-19 also increases the risk of thrombosis (13). The most important indicator of thromboembolic events that are already considered an important cause of mortality in the currently known pathogenesis of COVID-19 in the clinical follow-up is the increase in D-dimer. The D-dimer values increased the most in Group 3, i.e., in the group that was administered with pulse-steroid therapy during the 4-day follow-up in this study. This is the most important result of this study.

Despite the absence of a statistically significant difference, NLR values increased in all three groups while the lowest rate of increase was seen in Group 2 ( $p>0.05)$. The decrease in the CRP values in Group 2 and Group 3 are considered the most definite effect of corticosteroids. However, there are no major differences in the response to CRP suppression in terms of using low dose of steroid and pulse steroid treatment, while both doses suppress the CRP values to the same extent and time in clinical usage.

The fact that there was no significant difference between the groups in terms of the duration of hospital stay of surviving patients shows that pulse steroid treatment does not shorten the duration of hospital stay in patients severely or critically ill with COVID-19. The mortality rate and need for invasive or non-invasive ventilation were higher in Group 3 than in Group $2(p<0.05)$. Another remarkable result of this study is that the need for anti-cytokine treatment (tocilizumab or anakinra) after the first 4 days was found at a higher rate in the pulse steroid therapy group although a similar achievement of suppressing CRP was reached in both treatment groups $(\mathrm{p}<0.05$. The reason for this situation is that in this clinic, the decision for using pulse steroid treatment was generally taken in patients with higher basal CRP levels. This is the reason why the CRP levels were high among the patients who were administered with pulse-steroid treatment on the 1st day. A limitation of this study is that the homogenization was not sufficient among the groups in terms of CRP due to this administration. Therefore, it is normal that the need for anti-cytokine in the continuation of the treatment is higher in Group 3. Although bacterial reproduction 
was higher in Group 3 in the continuance of the treatment, this was not statistically different from the other groups $(p>0.05)$.

There are single-center studies and reviews with limited numbers of patients with different results regarding the corticosteroid treatment modalities applied to COVID-19 patients in the literature $(14,15,16,17)$. It can be indicated that the common features of these studies is stating the "need for more relevant studies", different doses of corticosteroids, and heterogeneous groups.

In the case series by Edalatifard et al including 68 patients, a group was administered with methylprednisolone therapy at a dose of $250 \mathrm{mg}$ /day for 3 days and the mortality rate was lower and recovery duration was shorter in this group as compared to the control group (18). The contradicting result with the current study is the decrease in the $\mathrm{D}$-dimer level in the methylprednisolone group. Although different doses of steroids were used for Group 2 and Group 3, there was an increase in D-dimer levels in both groups. The dose of corticosteroid used in the study by Edalatifard et al was between the doses of corticosteroid administered to the patients in Group 2 and Group 3 in this study. However, the patient group included in their study had some differences as compared to the present study. The patient groups in the study by Edalatifard et al consisted of patients in an early pulmonary involvement phase of COVID-19. The patients in the present study were more critically ill patients with pulmonary involvement that had already progressed at least into 4 lung lobes. Considering the effect of the severity of infection on the D-dimer level, it can be concluded that the changes in D-dimer in both studies are related to the patient population.

There are many studies on using D-dimer and NLR parameters for assessing the prognosis and predicting mortality in patients with COVID-19 in the literature, and both parameters have an important place in the follow-up of critical patients $(19,20)$. The D-dimer and NLR cut-off values were determined relative to mortality predictability, and the numbers of patients with values above these cut off values were analyzed in this study. Accordingly, the highest number of patients with values exceeding the cut off value determined for D-dimer $(2,250 \mathrm{ng} / \mathrm{mL})$ was in Group 1 while $52.5 \%$ of the patients in Group 3 and $32.5 \%$ of the patients in Group 2 had values above this cut-off value. The highest ratio of the patients with values exceeding the cut-off value determined for NLR belonged to Group 3. The results regarding both values used for the prognosis and mortality predictions were worse in the pulse steroid therapy group.

In the study by Mareev et al conducted on 34 patients, the steroid group received methylprednisolone for 3 days at a dose of $1 \mathrm{~g} /$ day and were maintained on dexamethasone at a dose of $8 \mathrm{mg} /$ day, and this group was compared with the control group who did not receive steroids (15). The results of this study using a dose similar to that used in Group 3, were similar to the present study. In the study by Mareev et al, there was a significant increase in D-dimer, NLR, neutrophil count and platelet count and a significant decrease in CRP in the corticosteroid group $(p<0.05)$. A significant difference was found between the groups in terms of the changes in creatinine, ferritin, CRP, D-dimer, and platelet count in this study $(p<0.05)$. It would not be appropriate to put an interpretation on creatinine due to the insufficient homogenization in the groups in terms of creatinine value. However, the analysis results obtained with other parameters mainly support the study by Mareev et al. It was also emphasized in their study that using high dose of corticosteroid increases the risk of thrombosis.

This study has some limitations. The first limitation is that this is a retrospective study. There might be a selection bias due to the retrospective design of the study. Another limitation is that the homogenization was insufficient in terms of creatinine and CRP among the groups due to the high number of parameters examined.

\section{Conclusion}

In conclusion, it has been found in this study that pulse steroid therapy does not shorten the duration of hospital stay, does not reduce the need for intubation and increases the risk of thrombosis by significantly increasing the level of D-dimer among patients hospitalized in the intensive care unit for being critically or severely ill with COVID-19. Although positive results were obtained with pulse-steroid treatment when administered in early pulmonary involvement of COVID-19, starting the pulse-steroid treatment in patients with a progressed pulmonary involvement needs to be seriously questioned. We do not recommend starting a pulse steroid treatment routinely in the patients severely ill with COVID-19 in ICU.

\section{References}

1. Gundogdu O, Avci O. Comparing the Clinical, Radiological and Laboratory Characteristics of Confirmed or Suspected COVID-19 Cases. Erciyes Med J 2021; 43 (2).

2. Sanders JM, Monogue ML, Jodlowski TZ, Cutrell JB. Pharmacologic Treatments for Coronavirus Disease 2019 (COVID-19): A Review. JAMA 2020; 323 (18): 1824-1836.

3. Goyal A, Saigal S, Niwariya Y, Sharma J, Singh P. Successful use of tPA for thrombolysis in COVID related ARDS: a case series. J Thromb Thrombolysis 2020; $1-4$.

4. Coperchini F, Chiovato L, Croce L, Magri F, Rotondi M. The cytokine storm in COVID-19: An overview of the involvement of the chemokine/chemokine-receptor system. Cytokine Growth Factor Rev 2020; 53: 25-32.

5. Sun X, Wang T, Cai D et al. Cytokine storm intervention in the early stages of COVID-19 pneumonia. Cytokine Growth Factor Rev 2020; 53 : $38-42$.

6. Tobaiqy M, Qashqary M, Al-Dahery S et al. Therapeutic management of patients with COVID-19: a systematic review. Infect Prevent Pract 2020; 100061.

7. Darmon P, Dadoun F, Boullu-Ciocca S, Grino M, Alessi M-C, Dutour A. Insulin resistance induced by hydrocortisone is increased in patients with abdominal obesity. Amer J Physiol Endocrinol Metab 2006; 291 (5): E995-1002.

8. Clore J, Thurby-Hay L. Glucocorticoid-Induced Hyperglycemia. Endocrine Pract 2009; 15 (5): 469-474.

9. Shono A, Mori S, Nakamura K et al. Glucocorticoid-sensitive Paroxysmal Atrial Fibrillation, Sick Sinus Syndrome, and Mitral Regurgitation 


\section{3-798}

in a Patient with Malignant Rheumatoid Vasculitis. Internal Med (Tokyo, Japan) 2019; 58 (21): 3093-3098.

10. Surmachevska N, Tiwari V. Corticosteroid Induced Myopathy. (Updated 2020 May 6). In: StatPearls (Internet). Treasure Island (FL): StatPearls Publishing; 2021 Jan.

11. Kayim Yildiz O, Yildiz B, Avci O, Hasbek M, Kanat S. Clinical, Neurophysiological and Neuroimaging Findings of Critical Illness Myopathy After COVID-19. Cureus 13 (3): e13807.

12. Ozsu, S, Gunay, E, Konstantinides, SV. A review of venous thromboembolism in COVID-19: A clinical perspective. Clin Respir J 2021: 1- 7.

13. Gupta N, Zhao YY, Evans CE. The stimulation of thrombosis by hypoxia. Thromb Res 2019; 181: 77-83.

14. Boonyawat, $K$, Chantrathammachart $P$, Numthavaj $P$ et al. Incidence of thromboembolism in patients with COVID-19: a systematic review and meta-analysis. Thrombosis J 2020; 18: 34.

15. Mareev VYu, Orlova YaA, Pavlikova EP et al. Steroid pulse -therapy in patients with coronavirus pneumonia (COVID-19), systemic inflammation and risk of venous thrombosis and thromboembolism (WAYFARER Study). Kardiologiia 2020; 60 (6): 15-29.
16. Cano EJ, Fonseca Fuentes X, Corsini Campioli C et al. Impact of Corticosteroids in Coronavirus Disease 2019 Outcomes: Systematic Review and Meta-analysis. Chest 2021; 159 (3): 1019-1040.

17. Dolci G, Cassone G, Venturelli F et al. High-dose glucocorticoids pulse-therapy for beta-coronaviridae pneumonia: a systematic literature review and case-series of Coronavirus disease-2019. Clin Exp Rheumatol 2021.

18. Edalatifard M, Akhtari M, Salehi M et al. Intravenous methylprednisolone pulse as a treatment for hospitalised severe COVID-19 patients: results from a randomised controlled clinical trial. Eur Respir J 2020; 56 (6): 2002808 .

19. Ye W, Chen G, Li X et al. Dynamic changes of D-dimer and neutrophil-lymphocyte count ratio as prognostic biomarkers in COVID-19. Respir Res 2020; 21: 169.

20. Ai-Ping Yang, Jian-ping Liu, Wen-qiang Tao, Hui-ming Li, The diagnostic and predictive role of NLR, d-NLR and PLR in COVID-19 patients. Internat Immunopharmacol 2020; 84: 106504.

Received April 25, 2021. Accepted May 4, 2021. 